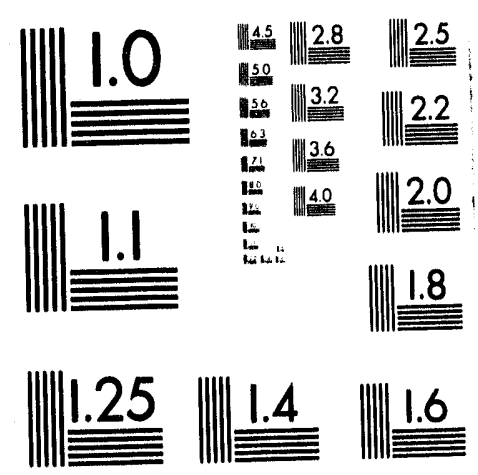



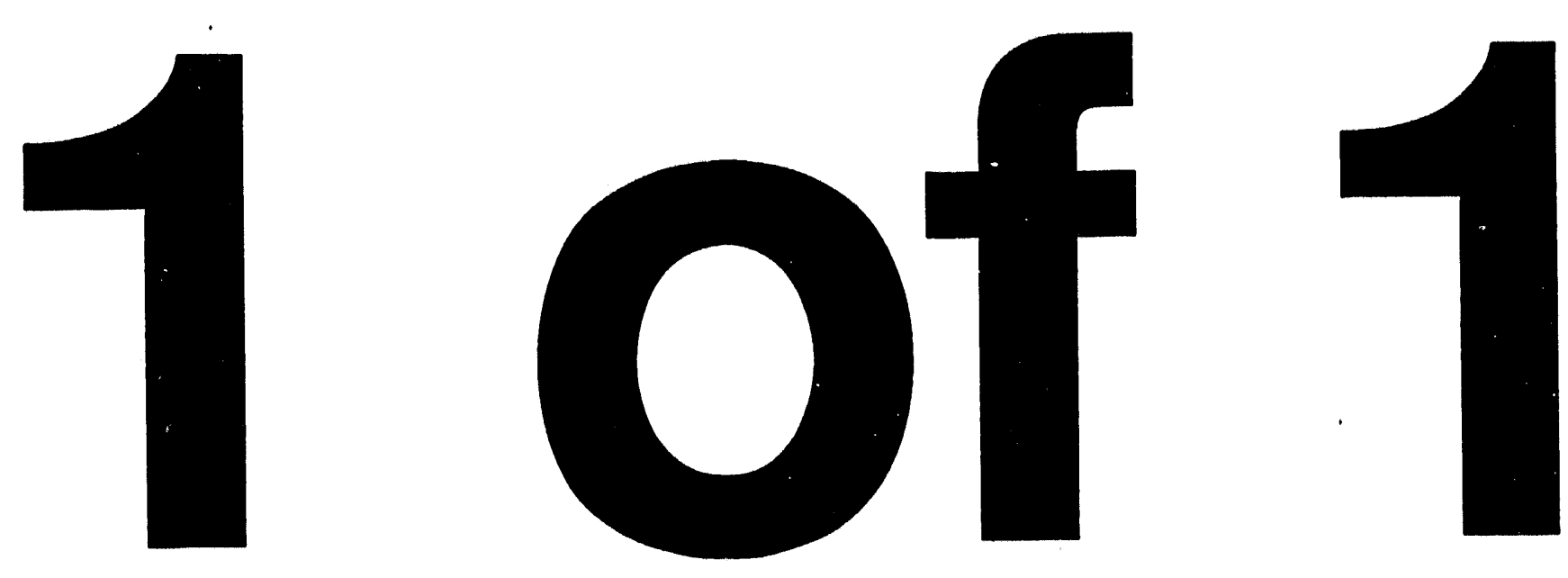
TITLE: A HIGH PERFORMANCE COMMUNICATIONS AND MEMORY CACHING SCHEME FOR MOLECULAR DYNAMICS ON THE CM-5

AUTHOR(S): N. Grønbech-Jensen, T-11

Peter S. Lomdahl, T-11

Pablo Tamayo, T-8

David M. Beazley, CNLS

SUBMITTED TO: 8 th International Parallel Processing Symposium

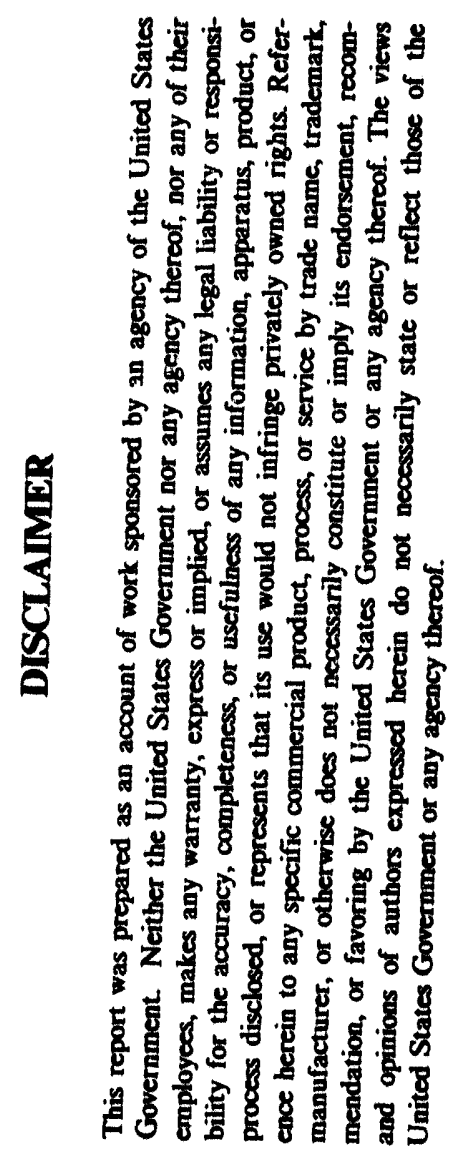

By acceptance of this article, the publisher recognized that the U S Government retains a nonexclusive, royalty-Iree license to publlsh or reproduce the published form of this contribution or to allow others to do so for U S Government purposes.

The Los Alamos National Laboratory requests that the publisher identify this article as work performed under the auspices of the U S Department of Energy.

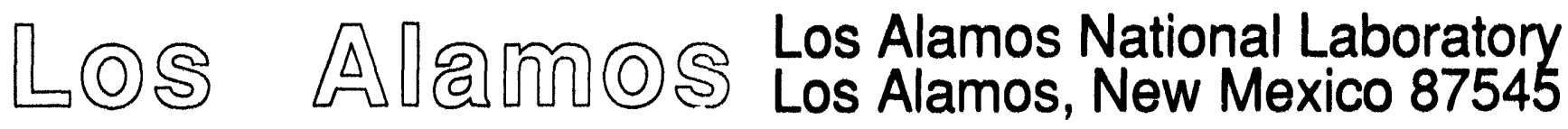




\title{
A High Performance Communications and Memory Caching Scheme for Molecular Dynamics on the CM-5
}

\author{
David M. Beazley, Peter S. Lomdahl, \\ Niels Grønbech-Jensen, and Pablo Tamayo \\ Theoretical Division and Advanced Computing Laboratory \\ Los Alamos National Laboratory, Los Alamos, NM 87545
}

September 15, 1993

\section{Introduction}

For several decades, the method of molecular dynamics (MD)[1] has been used to study the dynamical properties of solids and liquids. In an a molecular dynamics simulation, one wants to solve Newton's equations of motion for a large collection of $N$ interacting particles. This general $N$-body problem involves the calculation of $N(N-1) / 2$ pair interactions to compute the force on any given particle and for large systems of particles, this calculation is extremely complicated. To simplify the calculation, most MD simulations assume that each particle has a finite range of interaction and only interacts with particles that are nearby. While this greatly reduces the complexity of the problem, there are still many computational difficulties associated with short-range MD. Many MD algorithms have been implemented on variety of computing systems, but until recently most $\mathrm{MD}$ simulations have been limited to systems involving less than a million atoms[2, ?]. However, to perform realistic simulations in materials science, it is necessary to simulate hundreds of millions or even billions of atoms for many thousands of time steps. While billion atom simulations are still very far away there is considerable interest in developing fast $M D$ algorithms $[2,3,4,5,6,7]$. The rapid development of massively parallel supercomputers has allowed for the simulation of a greater number of particles than ever before. On existing hardware, simulations with more than 100 million $\left(10^{8}\right)$ particles can now be performed. It is clear that future advances in parallel computing may make the goal of simulating a billion particles a reality.

We have developed a scalable MD algorithm based on a message passing approach that allows for simulating at least $10^{8}$ particles interacting via a relative short-range potential. We have implemented the algorithm in a code, SPaSM (ㅇcalable Parallel Short-range Molecular dynamics), on the Connection Machine 5 (CM-5). Using this code, realistic simulations involving tens of millions of particles can be performed routinely. Originally, our code was developed on the CM-5 as a general message passing code that could be adapted to other 
message passing machines or a cluster of workstations. However, to obtain high performance on the CM-5, we have made several modifications that optimize communications and allow us to efficiently use the CM-5 vector units. These modifications allow our code to run more than 5 times faster than before with sustained calculation rates as high as 50 Gflops on a 1024 processor CM-5[8]. As a result, our code (SPaSM) is a finalist in the 1993 IEEE Gordon Bell prize competition. Our modifications rely heavily on CM-5 architecture, but we have been able to achieve high performance while still maintaining a high degree of code portability. These modifications are highly modular and can be removed or rewritten without having to change our MD algorithm. This will allow us to easily port our code to other message passing environments and future machines.

In this paper, we provide a brief overview of our general MD algorithm and focus on several performance enhancements that have allowed us to achieve high performance on the CM-5. Our use of the CM-5 vector units (VUs) to calculate forces is described along with a memory caching scheme that speeds up the force calculation by as much as $50 \%$. In addition, we discuss a method used to speed up the communication aspects of our algorithm by more than $35 \%$. Lastly, recent timing and scaling results are presented. Our code has been implemented in ANSI $\mathrm{C}$ with explicit calls to the CMMD message-passing library. To use the VUs we have written our force calculation in CDPEAC (a C interface to the $\mathrm{VU}$ assembler language, DPEAC). We also assume that particles interact according to the Lennard-Jones 6-12 (LJ) potential

$$
V(r)= \begin{cases}4 \epsilon\left(\left(\frac{\sigma}{r}\right)^{12}-\left(\frac{\sigma}{r}\right)^{6}\right) & 0<r \leq r_{\max } \\ 0 & r_{\max }<r\end{cases}
$$

Here $\sigma$ and $\epsilon$ are the usual LJ parameters. The potential is cut-off at, $r_{\max }$, i.e. no particles interact beyond this range. While this potential is quite common in MD simulations, we should stress that our algorithm can use other short-range potentials.

\section{The Multi-cell MD Algorithm}

Our algorithm has been described in detail in[7]. Here we briefly outline its main features, illustrating the algorithm in 2D, but it extends naturally to $3 \mathrm{D}$. The main problem in all short-range MD simulations is that of determining which particles interact and calculating the resulting forces. To address this problem, we consider space to be a rectangular region with periodic boundary conditions. This region is subdivided into large cells that are assigned to the processing nodes (PNs) on the CM-5. The region assigned to each $\mathrm{PN}$ is further subdivided into small cells with dimensions slightly larger than the cutoff distance $r_{\max }$. Particles are assigned to a particular cell geometrically according to the particle's coordinates. This layout allows us to quickly determine which particles interact since the interacting neighbors for any particular particle will be in the same cell or nearby cells. This also distributes the particles over all of the processing nodes available. In Fig. 1, solid lines represent processor boundaries while dashed lines represent the cells created on each PN. 


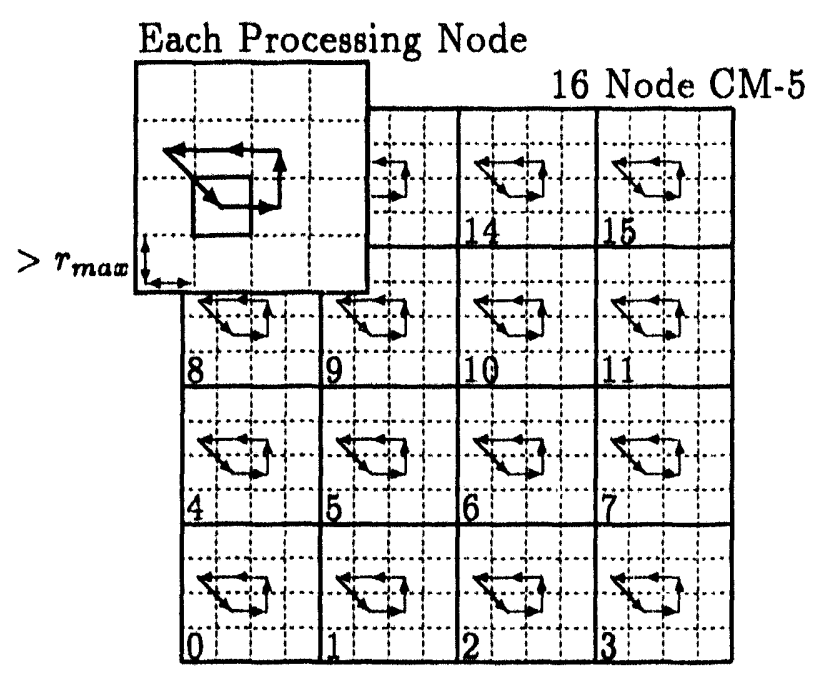

Fig. 1. Processor layout and force calculation.

For large simulations, many thousands of cells per PN may be created (this does not explicitly depend on the number of PNs being used).

Associated with each cell is a small block of memory for storing a sequential list of particles. Each particle is a $\mathrm{C}$ structure containing the position, velocity, force, and particle type. To compute the forces for the particles in a cell, we first compute all of the interactions between particles in the same cell. Afterwards, interactions with particles in neighboring cells are calculated by following an interaction path that visits neighboring cells. The path in 2D is shown in Fig. 1 and in 3D in Fig. 2. As we follow the path, accelerations are accurnulated by the original cell and any visited cells (using Newton's third law). To calculate all of the forces, this procedure is carried out on all cells on all of the PNs. Cells will accumulate accelerations from their lower neighbors when they calculate their interactions. Whenever the interaction path crosses a processor boundary, message passing is used to communicate particle data. After all forces have been calculated, the particle positions are updated. Since our algorithm is geometrically based, all of the data structures must be updated to account for positional changes. The particle coordinates are checked and if a particle is in the wrong cell it is moved to the proper cell. If the new cell is on a different PN, asynchronous message passing is used to send the particle to its new PN. Each PN checks for incoming particles and places them in the proper cell when received. After all data structures have updated, the algorithm proceeds to the next time-step and the process is repeated.

\section{Parallel Memory and Vector Units}

On the CM-5, each processing node consists of a SPARC processor and four vector units (VUs) that operate in a SIMD mode. Each VU has a peak speed of 32 MFlops for a combined speed of 128 MFlops per node. In addition to performing fast vector arithmetic, the VUs act 


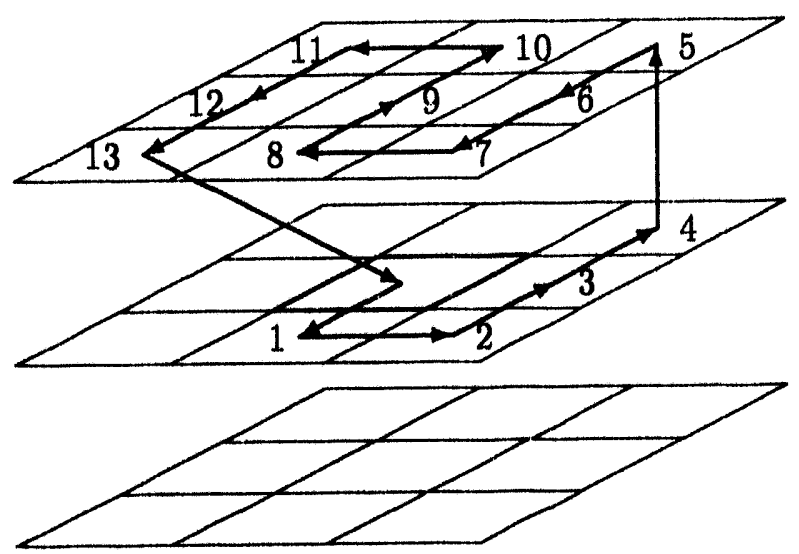

Fig. 2. 3D interaction path

as memory controllers where each VU controls an 8 MByte bank of memory for a total of 32 MBytes per node. The SPARC processor and the VUs share the memory on each PN, but memory is divided into two regions for each purpose. SPARC memory is memory that has been allocated to the SPARC processor and all usual memory operations perform normally. Parallel memory is a special memory allocation that allows the four VUs to participate in simultaneous load/store operations. Each VU allocates an identically structured block of memory in the 8 Mbyte block that they control. When loading or storing data, each VU uses its particular bank of memory. This allows the VUs to operate on four different data sets simultaneously. The amount of memory allocated to the SPARC processor and the vector units can be adjusted dynamically so it is possible for a program to use both types of memory as necessary. Physically, SPARC memory and parallel memory are part of the same RAM, but the virtual addresses used to access each region are calculated differently. For this reason, the two memory regions are considered as separate. Although the two memory regions are separated, data can be transferred between the two areas. The SPARC can access any particular bank of parallel memory, but accessing SPARC memory from the VUs can not be done without special instructions. As a result, accessing SPARC memory from the VUs is slow and should be avoided. As a general rule, all operations involving the VUs must use parallel memory to achieve optimal performance.

\subsection{Calculating Accelerations}

When calculating the accelerations for the particles in each cell, we follow an interaction path determines that the order in which the interactions with particles in neighboring cells are calculated. At each step of the interaction path, the accelerations between two cells of particles must be calculated. To efficiently calculate accelerations using the vector units, a force kernel has been written in CDPEAC. The kernel takes the lists of particles in two cells and calculates all of the resulting accelerations. In Fig. 3 this calculation is described. 


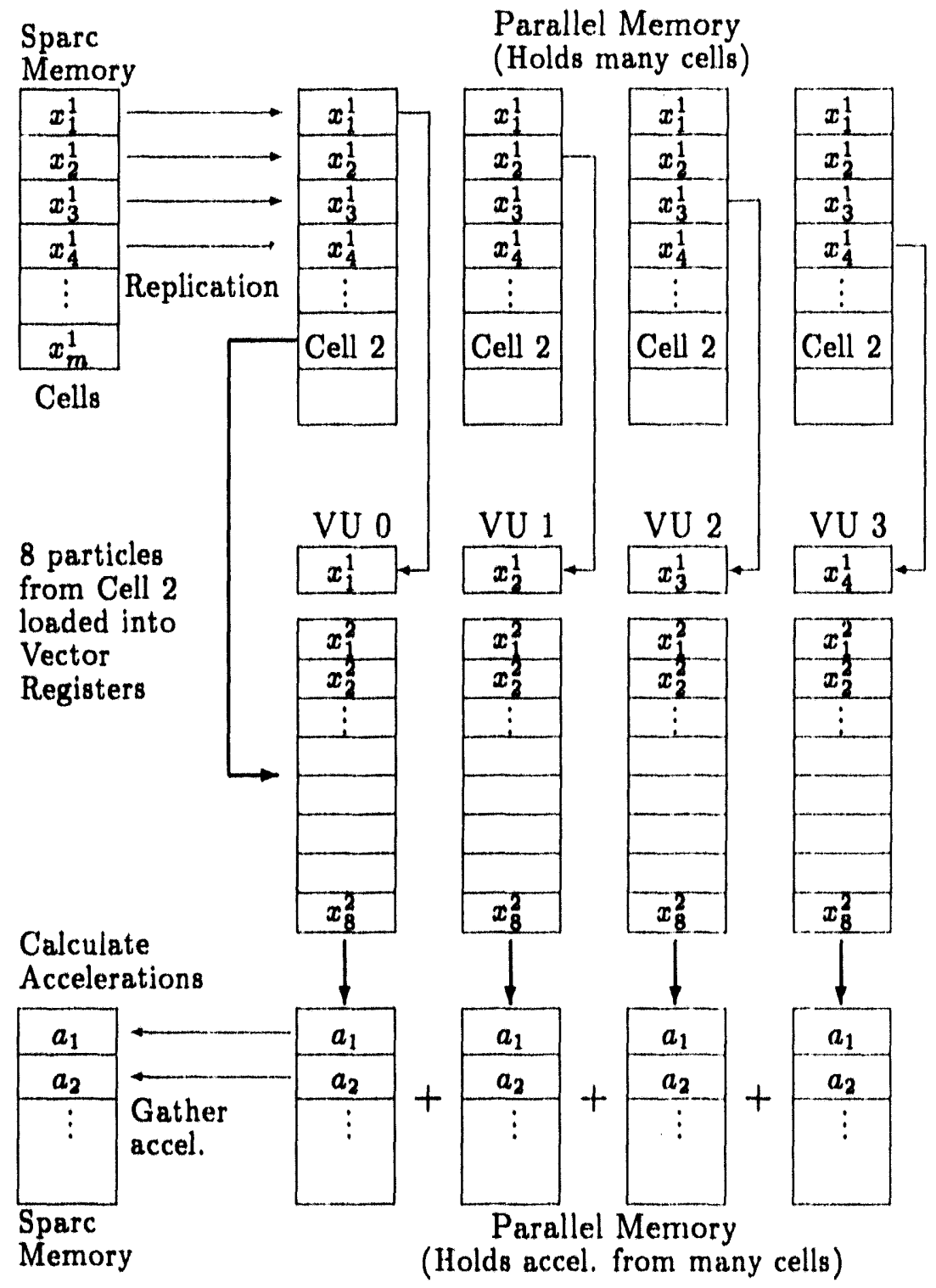

Fig. 3. Calculating forces on the VUs

The calculation begins by replicating the particle coordinates from both lists across the four vector units in parallel memory. This generates four identical lists of particles in parallel mernory (one list in each bank). After the coordinates are loaded, 8 particles from cell 2 are loaded onto the VUs. We then loop through all of the particles in cell 1 and calculate the accelerations between these particles and the 8 particles loaded from cell 2. At each step, memory indirection (supported by special CDPEAC instructions) is used to load four different particles from cell 1 onto the the VUs (a different particle on each VU). This allows the VUs to calculate 32 interactions at each step. Once all of the particles in cell 1 have been processed, the next set of 8 particles from cell 2 is loaded and the process is repeated. The calculation of forces continues, calculating 32 interactions per step, until all forces have 
been calculated. Afterwards, the resulting accelerations are gathered from parallel memory and saved back to SPARC memory for future use.

\subsection{Using Parallel Memory}

All internal data structures in our code are kept in SPARC memory. This has the advantage of the data being easily accessible to functions that make no use of the VUs. This includes numerical integrators, I/O functions, energy calculations, and any functions that involve message passing. It is also generally easier to organize and maintain data when it is in a single bank of memory instead of distributed across four banks of parallel memory. However, our use of the vector units requires particle data to be copied to parallel memory before any forces can be calculated. This transfer of data between SPARC and parallel memory can degrade performance so our goal is to minimize the time spent transferring data between the two memory regions. By minimizing the transfer time, we can maximize our use of the VUs while still keeping our data in SPARC memory.

One solution to the parallel memory problem is to simply copy particle coordinates to parallel memory whenever needed in the force calculation. With this approach, only two cells would be loaded in parallel memory at any given time. The method requires a minimal amount of purallel memory and is reasonably efficient if each cell has a large number of particles (since most time will be spent in the force kernel). Unfortunately, each cell may have to be loaded to parallel memory as many as 14 times (once when calculating selfinteractions and 13 times when neighboring cells calculate their interactions). This excessive loading creates a bottleneck in the code and significantly degrades performance when each cell has a relatively small number of particles. In this case, it becomes difficult to keep the vector units busy since each PN may spend as much as $30 \%$ of its processing time doing nothing but transferring data between parallel and SPARC memury.

To eliminate the problem of excessive loading, a second apprcach is to load all of the particle coordinates to parallel memory at the beginning of the force calculation. This provides the best possible performance of our algorithm since the particles in each cell are always available in parallel memory for the force calculator. In some cases, this technique speeds up the force calculation by as much as $50 \%$, reducing the loading time to only a few percent of the overall calculation time. Unfortunately, this approach is extremely wasteful of memory since all of the particle coordinates must be replicated across all four VUs. This results in a memory overhead nearly $400 \%$ larger than the amount of memory required to store the particles in SPARC memory. This overhead is prohibitively expensive for even moderately sized systems and clearly prevents runs with many millions of particles.

\section{Parallel Memory Caching}

To solve the problem of transferring data between SPARC and parallel memory, we have developed a parallel memory caching scheme. This method provides the speed of loading all of the cells to parallel memory while saving a large amount of memory. 


\subsection{A Caching Scheme}

The caching scheme begins by allocating a buffer for holding cells in parallel memory. The first time each cell is encountered in the force calculation, it is loaded into this buffer. The loading operates according to a modified FIFO scheme and eventually new cells will begin to replace previously loaded cells. Each time a cell is encountered in the force calculation, this buffer is checked to see if that cell has already been loaded. If so, the particle coordinates are immediately available for computation. If not, the particles coordinates from the cell are loaded into the cache and the accelerations from any previously loaded cell saved back to SPARC memory. Two slight modifications are made to the FIFO loading scheme to improve performance and to prevent calculation hazards (in some cases the combination of caching and message passing can generate incorrect results without these modifications). The force calculator takes a particular cell, that we refer to as the home cell, and calculates the interactions between the home cell and its 13 neighboring cells by following the interaction path. In our caching scheme, we prevent the home cell from being removed from the cache while calculating interactions with its neighbors. This prevents the home cell from inadvertantly being removed from the cache and having to be immediately reloaded again to continue the force calculation. The interaction calculation also involves message passing with neighboring processors. Whenever particles are received from another processor, they will always have to be loaded to parallel memory. Since each PN will have at most one cell of "foreign" particles at any given time, we load these particles to a special buffer at the end of the cache area. This prevents the cache memory from being filled up with particles from other processors. The memory layout of the cache is shown in Fig. 4.

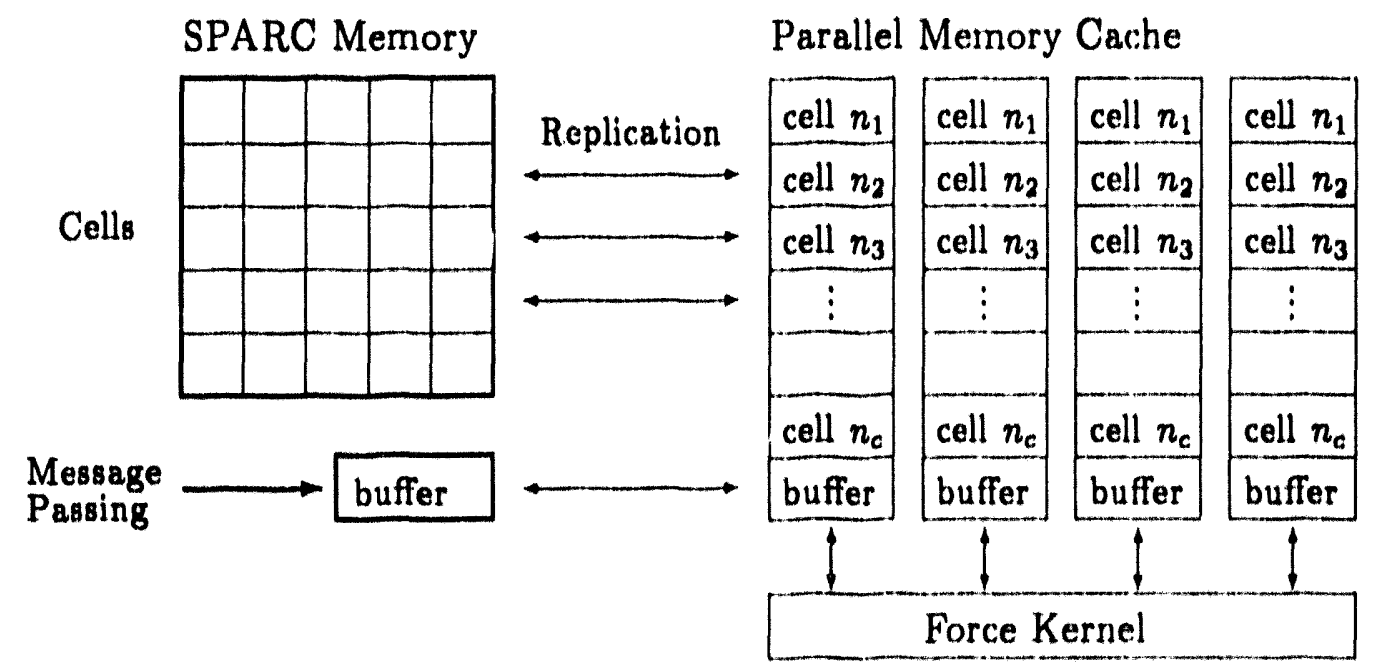

Fig. 4. Parallel Memory Caching Layout

The contents of the cache are replicated across the four banks of parallel memory. In the figure, $n_{c}$ represents the number of cells in the cache. The cached cells may correspond to any of the cells stored in SPARC memory. The cache acts as an interface between SPARC memory and the force kernel since the kernel operates only with data stored in the cache 
and has no knowledge of the data layout in SPARC memory. The double arrows indicate that data is exchanged in both directions. Particle coordinates are transferred from SPARC memory to the force kernel, while accelerations are transferred from the force kernel back to SPARC memory. Data transfer back to SPARC memory only occurs when a cell is removed from the cache.

The main idea of the caching scheme is that each cell is kept in parallel memory for as long as possible. At any time during the force calculation, only a small fraction of the total number of cells need to be loaded in parallel memory. This is due to the fact that the interaction path has a limited range and is illustrated for 2D in Fig. 5. In the figure, we process cells by rows starting at the bottom. A cache consisting of 2 rows of cells has been placed in parallel memory (indicated by the darkly shaded cells). As the calculation proceeds, we save old cells back to SPARC memory (lightly shaded cells), but these cells are now no longer necessary in the calculation since the path will not pass through these cells again. This is shown in Fig. $5 \mathrm{~b}$ and $5 \mathrm{c}$. Since our algorithm involves message passing on the boundaries, the cells that are cached can be slightly complicated as shown. In any case, most cells only need to be loaded to parallel memory once, providing optimal performance. in Fig. 5d, we see a slight complication that occurs when the upper boundary is reached. In this case, message passing occurs and it is necessary to calculate interactions involving the first row of cel!s again. Since these cells have been removed from the cache, they must be reloaded to pacallel memory again. As a result, only a small number of cells will have to be loaded to parallel memory more than once.

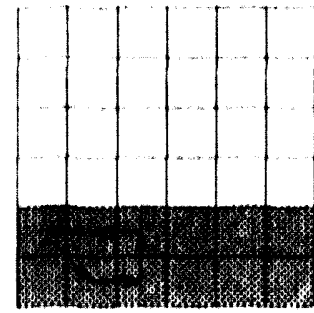

(a)

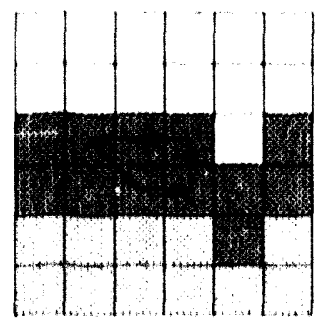

(c)

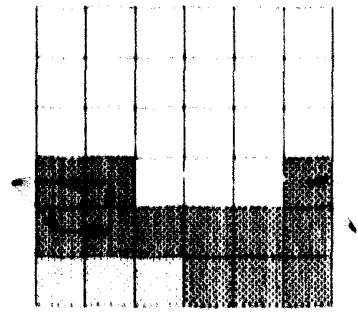

(b)

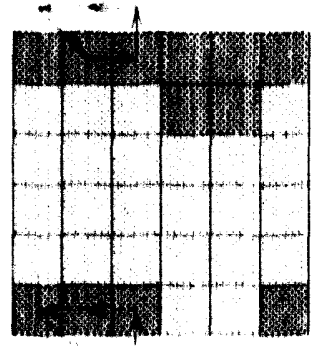

(d)

Cached Cells

Saved Calls

Figure 5. Caching at different stages of force calculation 


\subsection{Timing and Scaling Properties}

In our code, SPaSM, we can adjust the size of the cache. This allows us to optimize the code for both speed (using a large cache) or memory (using a small cache). Fig. 6, shows how the time required for a single timestep improves as we increase the number of cells that are cached. In the figure, a simulation with 8,192,000 particles was run on a 1024 PN CM-5. Each processor had 512 cells arranged in an $8 \times 8 \times 8$ configuration. With no caching, we pay a significant penalty for loading data in and out of parallel memory, but with even a slight increase the cache size, the time drops rapidly. Once $25 \%-30 \%$ of the cells have been cached, no further performance can be achieved by increasing the cache size. This allows us to obtain maximum performance with only $25 \%$ of the memory required for loading all of the cells to parallel memory. In the figure, the iteration time drops from approximately 4.1 seconds to 2.8 seconds as more cache is added. This represents a speedup of approximately $30 \%$. To better illustrate the effectiveness of caching, one can count the number of times data must be loaded to parallel memory (including data received during message passing). For the simulation in Fig. 6, using no caching requires 7741 parallel memory loads (where each load represents a single cell being copied to parallel memory). Caching only 20 cells reduces this to 3422 loads and caching 50 cells drops this to 1776 loads. After caching approximately 180 cells the iteration time levels out and 1149 loads are required. This represents a decrease of nearly $85 \%$ ! It should be noted that a slight speedup can be made by caching all of the cells. In this case it is not necessary to reload the cells along the initial boundary and only 1085 parallel memory loads are required. However, the extra performance gained is not worth the massive amount of memory required. We have found the caching scheme to be extremely effective with very large runs. In a simulation with 131 million $f$ articles and no caching, the iteration time was 54 seconds. By caching only $3 \%$ of the cells, the iteration time dropped by nearly $30 \%$ to 38.3 seconds.

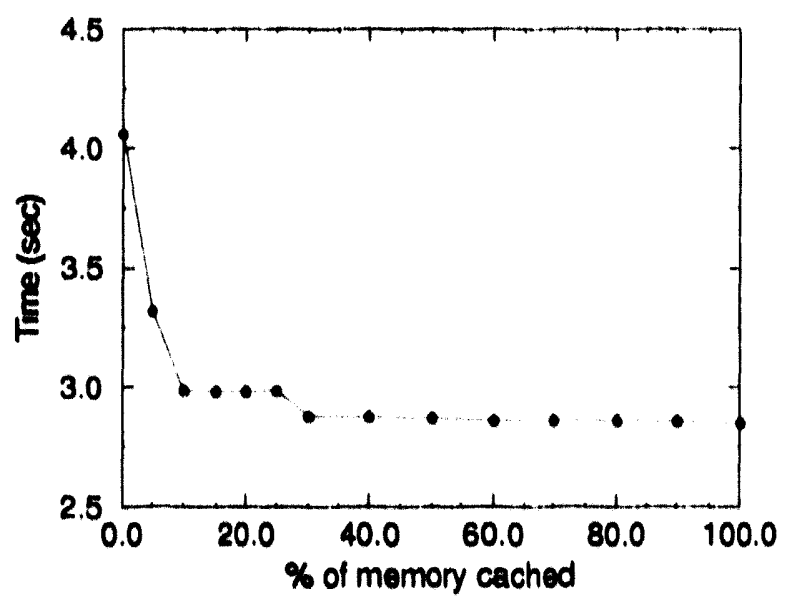

Fig. 6. Scaling of iteration time by cache size 


\subsection{Performance Considerations}

The use of parallel memory caching dramatically improves the speed of our code, but there are several aspects of our algorithm, indirectly related to the caching, that can be modified to improve the memory requirements of caching. Ideally, we want to obtain the best speedup possible with the smallest amount of parallel memory. This depends on both the geometry of cells and the order in which cells are processed.

For optimal performance, the cache must be large enough to store several layers of cells that completely contain the interaction path used. In Fig. 7, two different configurations of a systern are shown. In each case, cells are processed by rows from bottom to top.
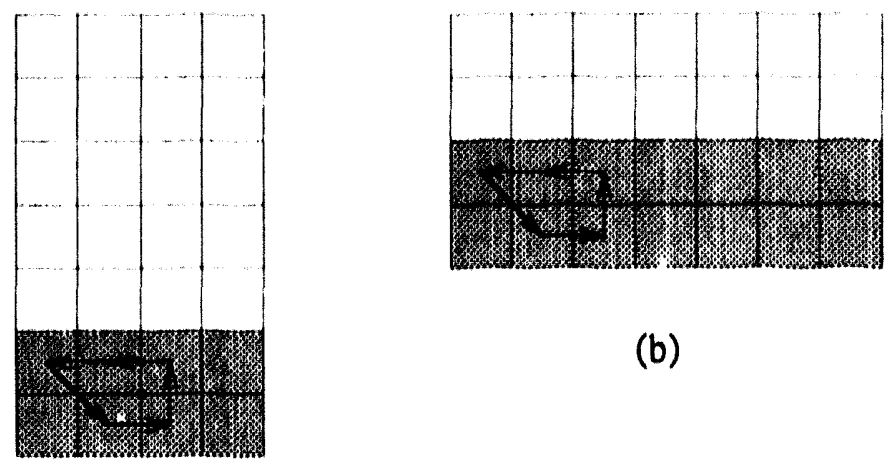

(b)

(a)

Fig. 7. Caching with different geometries

In Fig. 7a, optimal performance is obtained by caching only 8 cells, but if the same system is rotated by 90 degrees as in Fig. 7b, 14 cells will have to be stored in the cache. Clearly the best choice in this case is Fig. 7a. It will require less cache memory and will have better overall performance. In addition, we could double the height of the system in Fig. $7 \mathrm{a}$ without having to increase the cache size. Doubling the width in Fig. $7 \mathrm{~b}$ would require increasing the cache to 28 cells. For a given simulation, one should be careful to choose the geometry of the systern accordingly since this can have a dramatic effect on memory requirements.

Closely related to the geometry is the order in which cells are processed (by rows or columns for instance). Again, one wants to choose an order that yields the smallest possible cache. In Fig. 8, two different orders of processing cells are shown.

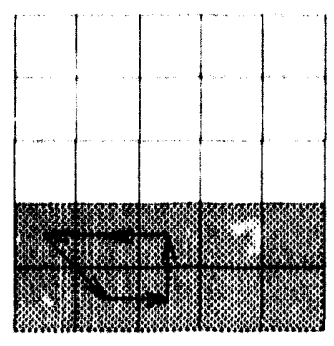

(a)

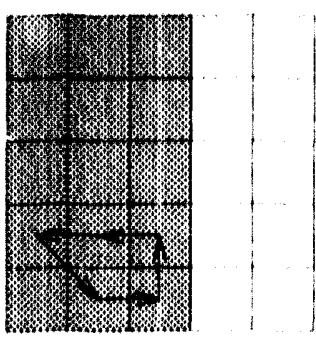

(b)

Fig. 8. Caching with different cell ordering 
In Fig. 8a, cells are processed by rows and only 2 rows need to be stored. In Fig. $8 \mathrm{~b}$, cells are processed by columns and 3 columns must be stored. To determine which order to use, one must look at the interaction path. Our 2D path is 3 columns wide and 2 rows deep. Since the path involves fewer rows, cells should be processed by rows. In 3D, the path is 3 cells across in both the $x$ and $y$ directions, but only 2 cells deep. In this case, we should process cells by horizontal layers starting from the bottom face.

\subsection{Implementation}

In our code, a general procedure is used to calculate all the interactions between particles. This procedure manages the path and order in which processing is performed. Whenever the actual forcés need to be calculated between cells, a single function call is made to a "force" function. The force function is isolated from the rest of the code, so there is a great deal of flexibility in writing a force calculation. All of our use of the VUs and parallel memory has been written into this single function. All other functions have no knowledge of parallel memory or the vector units (except for a few initialization calls that can be easily be removed). In this respect, the parallel memory caching scheme and force kernel operate entirely in the background and could be easily removed if the code were to be run on different parallel architectures. This gives our code better portability since we have been able to use the vector units of the CM-5 to achieve high performance without having to rewrite our entire algorithm.

\section{Minimizing Communication}

The use of an interaction path has been used previously in other MD algorithms (particularly on SIMD machines) and is an effective technique for calculating the forces between particles $[2$, 3]. In our particular algorithm, following the path requires no communications for most cells since each PN may contain many thousands of cells and only a fraction of these lie on processor boundaries. However, whenever the path does cross a processor boundary, message passing is used to transmit particle data. When using a path, it is very typical to chose a single interaction path and use it for all of the cells in the calculation (like a communications stencil). Unfortunately, we have found this approach to be somewhat inefficient since the chosen path may perform unnecessary communication across certain processor boundaries. In Fig. 9, two different 3D interaction paths are shown. 


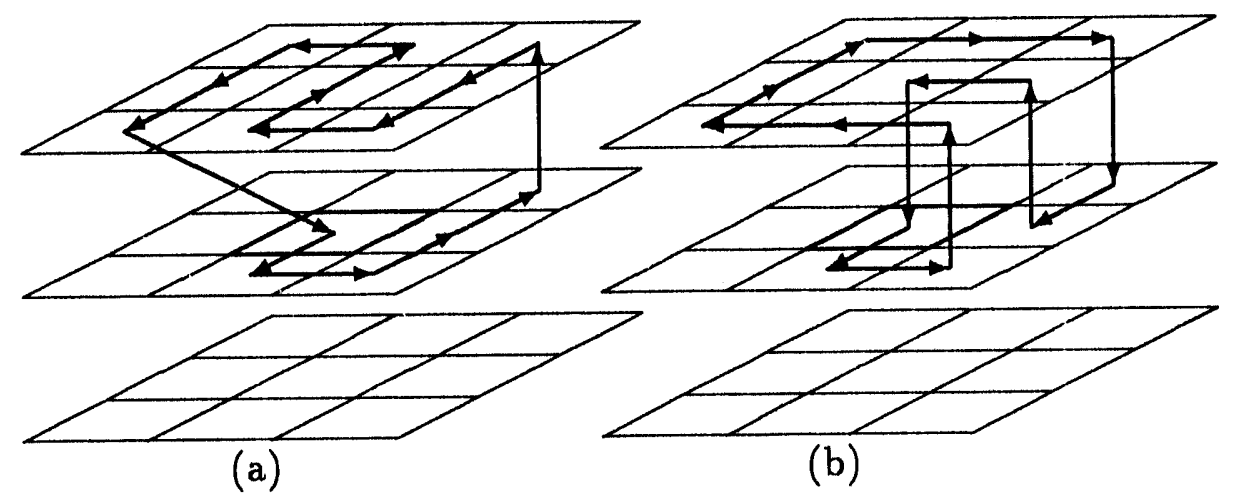

Fig. 9. Two different $3 D$ interaction paths

If path (a) is used, cells on the left or right sides of the processor space will require only 2 message passing calls while cells on the front face will require 6 calls. This is due to the fact that path (a) moves back and forth in the $x$ direction and will cross through the front face several times. If path (b) is used, unnecessary communication through the front and back face is eliminated, but now the path does not work well with the left and right sides.

Rather than use a single interaction path for all of the cells, we have found that excessive loading can be completely eliminated if we allow each cell to have a different interaction path. The only restriction is that each path must pass through the exact same set of neighboring cells (in order for Newton's third law to work). With this approach, we could assign path (a) to cells on the left and right sides and path (b) to cells on the front and back faces of processor space. Additional paths can be devised to minimize communications for edges and corners.

To illustrate the effectiveness of allowing multiple paths, suppose each processor has a $c_{x} \times c_{y} \times c_{z}$ grid of cells. If path (a) is used for all of the cells, the number of message passing calls required for each time step is given by

$$
M_{1}=2 c_{x} c_{y}+4 c_{x} c_{z}+10 c_{y} c_{z}-c_{x}-c_{y}-c_{z}+1
$$

This path is highly inefficient for cells in the $y z$-plane as indicated by the $10 c_{y} c_{z}$ term. If we allow multiple paths and eliminate all unncessary communications, the number of message passing calls can be reduced to the minimum number given by

$$
M_{n}=2 c_{x} c_{y}+4 c_{x} c_{z}+4 c_{y} c_{z}-c_{x}+5
$$

In this case, the number of message passes through the $y z$-plane has dropped by $60 \%$. In one simulation involving an $8 \times 8 \times 8$ grid of cells, using only path (a) resulted in 1001 message passing calls per time-step. Adding path (b) to the front and back faces reduced this to 698 message passing calls. Adding additional paths to the edges and corners reduced the amount of message passing even further to $637-\mathrm{a}$ savings of nearly $37 \%$ over using a single path. In our current code, 9 different paths are used to obtain the minimal amount of communication required for each time step. This has significantly reduced the communications time required 
by our algorithm. Optimizing communications also improves the performance of parallel memory caching since this reduces the number of times received particles must be copied to parallel memory. It should be noted that our optimization of communications does not depend on the CM-5 architecture and would improve the performance on other message passing machines.

We would also like to point out that our approach to communications is quite different than what is used in most MD algorithms proposed for MIMD machines. In many algorithms it is common for a processor to send all of the coordinates from particles on its boundaries to neighboring processors at the beginning of the force calculation[5]. This can be done in as few as 6 message passing calls in some cases[6]. However, these data exchanges typically involve large amounts of data and require a substantial buffer on each processor for storing all of the received particles. In addition, each processor must perform extra operations to determine which particles (and neighbor lists in some cases) must be sent to its neighbors. These particles must be gathered and stored in a buffer before any communications can occur. In our algorithm, we send one cell at a time which requires only a small receiving buffer on each processor. Message passing involves relatively small data packets and occurs periodically throughout the force calculation. Due to our data layout, we do not need to check particle coordinates or reorganize data before communication. While our algorithm may require many thousands of message passing calls for every time-step, we achieve both memory efficiency and performance since our algorithm requires only a small receiving buffer and does perform any unnecessary data management prior to communication. For this reason, our algorithm with optimized communications is competitive with other schemes to reduce the amount of communication.

\section{Timing Results}

In Table 1, recent timings for runs with a cutoff of $r_{\max }=2.5 \sigma$ are presented. The time required for one timestep and the update time per particle in $\mu$ sec is given. All runs were performed using parallel memory caching and optimized communications. In each case, $25 \%$ of the cells were cached except for the run with 131 million particles that used a $3 \%$ cache. Initially, the particles were arranged in a uniform $3 \mathrm{D}$ lattice at constant density $\rho=N / \sigma^{3}=1$ and given an initial velocity to ensure realistic interprocessor communications. With this choice of density and cutoff $r_{\max }=2.5 \sigma$, each particle will have approximately 65 interacting neighbors.

From the table we see that the algorithm scales nearly linearly with the number of particles and the number of processors. Scaling by the number of processors is shown in Fig. 10. Our best timing is that for 65 million particles on $1024 \mathrm{PNs}$. The update time of 16.55 seconds corresponds to 250 nano-seconds per particle. To the best of our knowledge, this is the best reported timing to date[6]. 


\begin{tabular}{|r|rrrrrr}
\hline & \multicolumn{6}{|c|}{ Processors } \\
Particles & 32 & 64 & 128 & 256 & 512 & 1024 \\
\hline 1024000 & $8.90(8.69)$ & $4.51(4.40)$ & $2.32(2.26)$ & $1.26(1.23)$ & $0.72(0.70)$ & $0.44(0.43)$ \\
2048000 & - & $8.96(4.38)$ & $4.44(2.17)$ & $2.46(1.20)$ & $1.36(0.66)$ & $0.74(0.36)$ \\
4096000 & - & - & $8.79(2.15)$ & $4.81(1.17)$ & $2.67(0.65)$ & $1.36(0.33)$ \\
8192000 & - & - & $16.83(2.05)$ & $8.81(1.08)$ & $4.80(0.59)$ & $2.47(0.30)$ \\
16384000 & - & - & - & $16.95(1.03)$ & $8.74(0.53)$ & $4.49(0.27)$ \\
32768000 & - & - & - & - & $16.90(0.52)$ & $8.54(0.26)$ \\
65536000 & - & - & - & - & - & $16.55(0.25)$ \\
131072000 & - & - & - & - & - & $34.26(0.26)$ \\
\hline
\end{tabular}

Table 1: Time for one time step in sec. (Time per particle update in $\mu \mathrm{sec}$ in parenthesis). Cut-off: $r_{\max }=2.5 \sigma$.

\begin{tabular}{|r|rrr|}
\hline Particles & Computation & Communications & Parallel Memory \\
\hline 4096000 & $70.7 \%$ & $23.8 \%$ & $5.5 \%$ \\
16384000 & $79.3 \%$ & $15.2 \%$ & $5.5 \%$ \\
65536000 & $84.1 \%$ & $10.5 \%$ & $5.4 \%$ \\
131072000 & $81.2 \%$ & $9.2 \%$ & $9.6 \%$ \\
\hline
\end{tabular}

Table 2: Timing breakdown for 1024 PNs. Cut-off: $r_{\max }=2.5 \sigma$

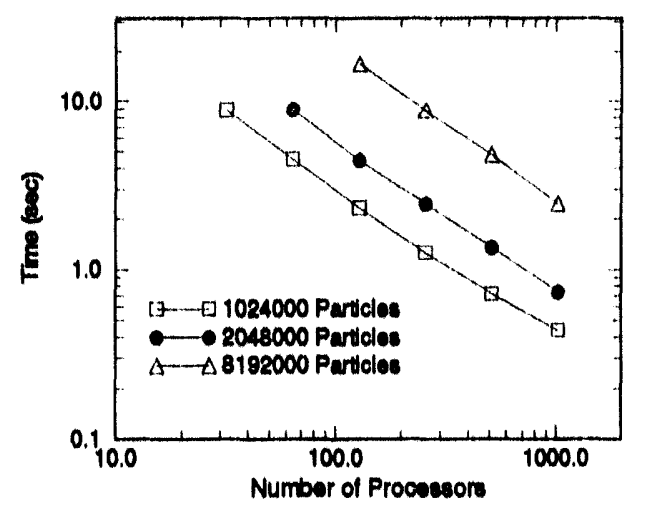

Fig. 10. Scaling by number of processors

In Table 2, the breakdown of communications, computation, and parallel memory access time is given. The algorithm is dominated by the calculation of forces. Parallel memory time is also low indicating the effectiveness of the parallel mernory caching scheme. The parallel memory loading time is larger for 131 million particles since a smaller cache size was used. The small amount of time spent loading parallel memory indicates that little is to be gained by further modifying our algorithm to store all of its data in parallel memory. In other tests, we were recently able to simulate 180 million particles in $3 \mathrm{D}$ with an 
update time of 55.6 seconds on a 1024 node CM-5. This run used minimal parallel memory caching, but represents the size system that can be simulated on state of the art parallel computers.

\section{Conclusion}

We have demonstrated that several enhancements can be made to our general MD algorithm to significantly improve performance. Each enhancment has been implemented at different stages of code development and this has allowed us to measure the improvement in performance. Our first version of code did not use vector units and a simulation involving 65 million 3D particles required approximately 87.4 seconds per time step[7]. To use the vector units, a force kernel was implemented in CDPEAC without caching and this reduced the iteration time to 36.2 seconds. To further improve our use of the VUs, we implemented a parallel memory caching and this reduced the iteration time to 19.1 seconds. Lastly, we optimized the communication aspects of our algorithm and this dropped the iteration time to the 16.55 seconds reported in this paper. As a result of the modifications, our code now runs over 5 times faster than before, but our general MD algorithm has changed little. Our use of the VUs and memory caching is highly modular and did not require major modifications to other parts of our code. This will make it easier to port the code to future machines that have entirely different architectures than the CM-5. It is interesting to note that the "recommended" way to use the CM-5 vector units has been write code in data-parallel CMF or $\mathrm{C} *$ since these languages work directly with parallel memory. However as a result of our parallel memory caching scheme, we have found that we only pay a ininimal performance penalty by just ignoring parallel memory and writing a standard $\mathrm{C}$ code that uses SPARC memory for storing its data structures. This provides our code both speed and portability and perhaps this approach can improve the performance of other $\mathrm{C}$ codes on the CM-5.

\section{Acknowledgements}

We thank the Advanced Computing Laboratory for generous support, in particular M. Krogh and D. Rich provided major assistance. We also express our appreciation to D. Dahl, A. Greenberg, C. Lobron, A. Mainwaring, and L. Tucker from TMC for valuable suggestions regarding CDPEAC and CMMD.

\section{References}

[1] Computer Simulations of Liquids, M. P. Allen and D. J. Tildesley. Clarendon Press, Oxford (1987).

[2] A. I. Mel'čuk, R. C. Giles, and H. Gould, Computers in Physics, May/June 1991, p. 311. 
[3] P. Tamayo, J. P. Mesirov, and B. M. Boghosian, Proc. of Supercomputing 91, IEEE Computer Society (1991), p. 462.

[4] B. L. Holian et al. Phys. Rev. A 43, 2655 (1991).

[5] R. C. Giles and P. Tamayo, Proc. of SHPCC'92, IEEE Computer Society (1992), p. 240.

[6] S. Plimpton and G. Heffelfinger, Proc. of SHPCC'92, IEEE Computer Society (1992), p. 246.

[7] D. M. Beazley and P. S. Lomdahl, Message-Passing Multi-Cell Molecular Dynamics on the Connection Machine 5, Parall. Comp. (1993) (in press).

[8] P. S. Lomdahl, P. Tamayo, N. Grønbech-Jensen, and D. M. Beazley, Proc, of Supercomputing 93, IEEE Computer Society (1993), (in press). 

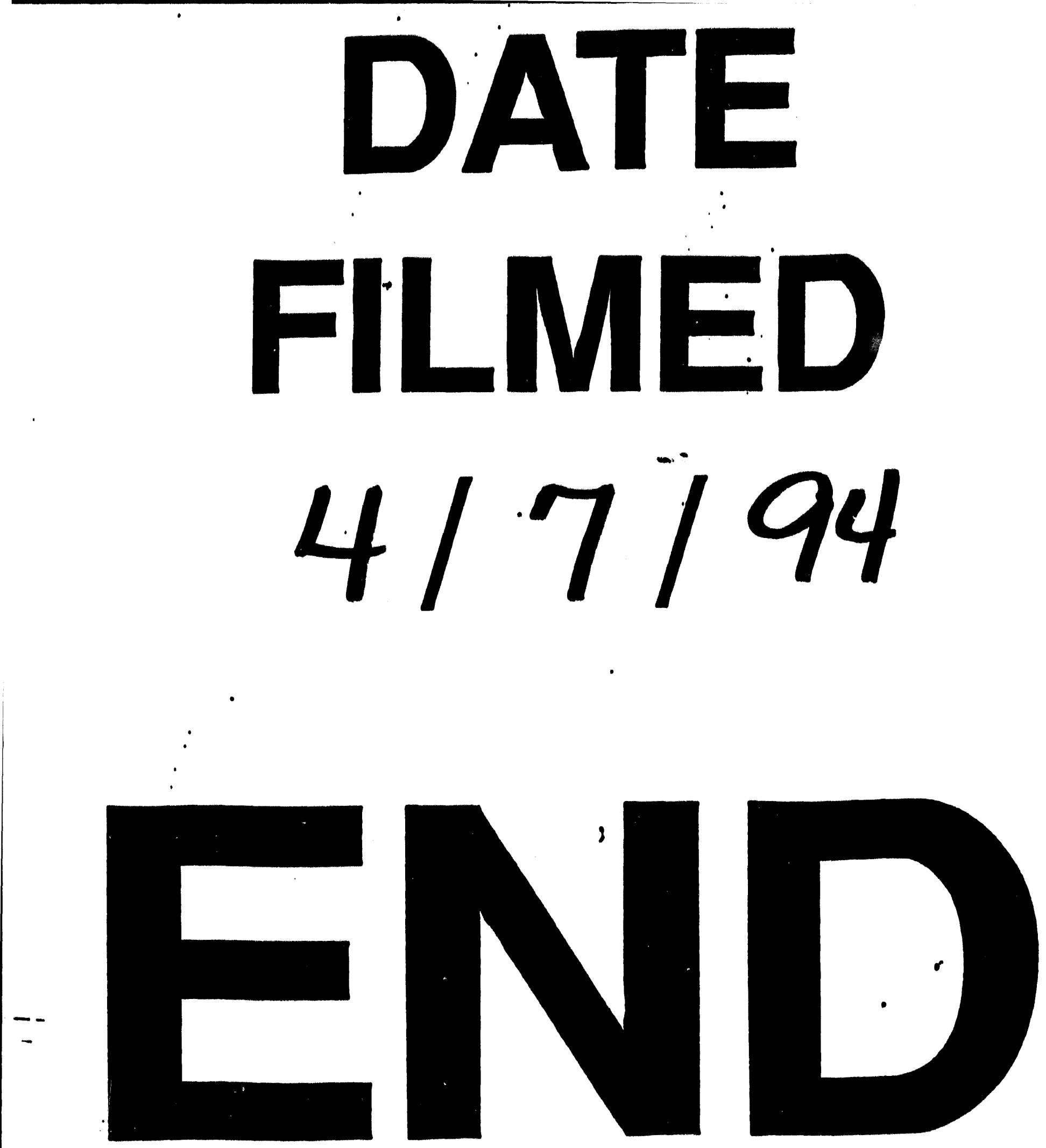


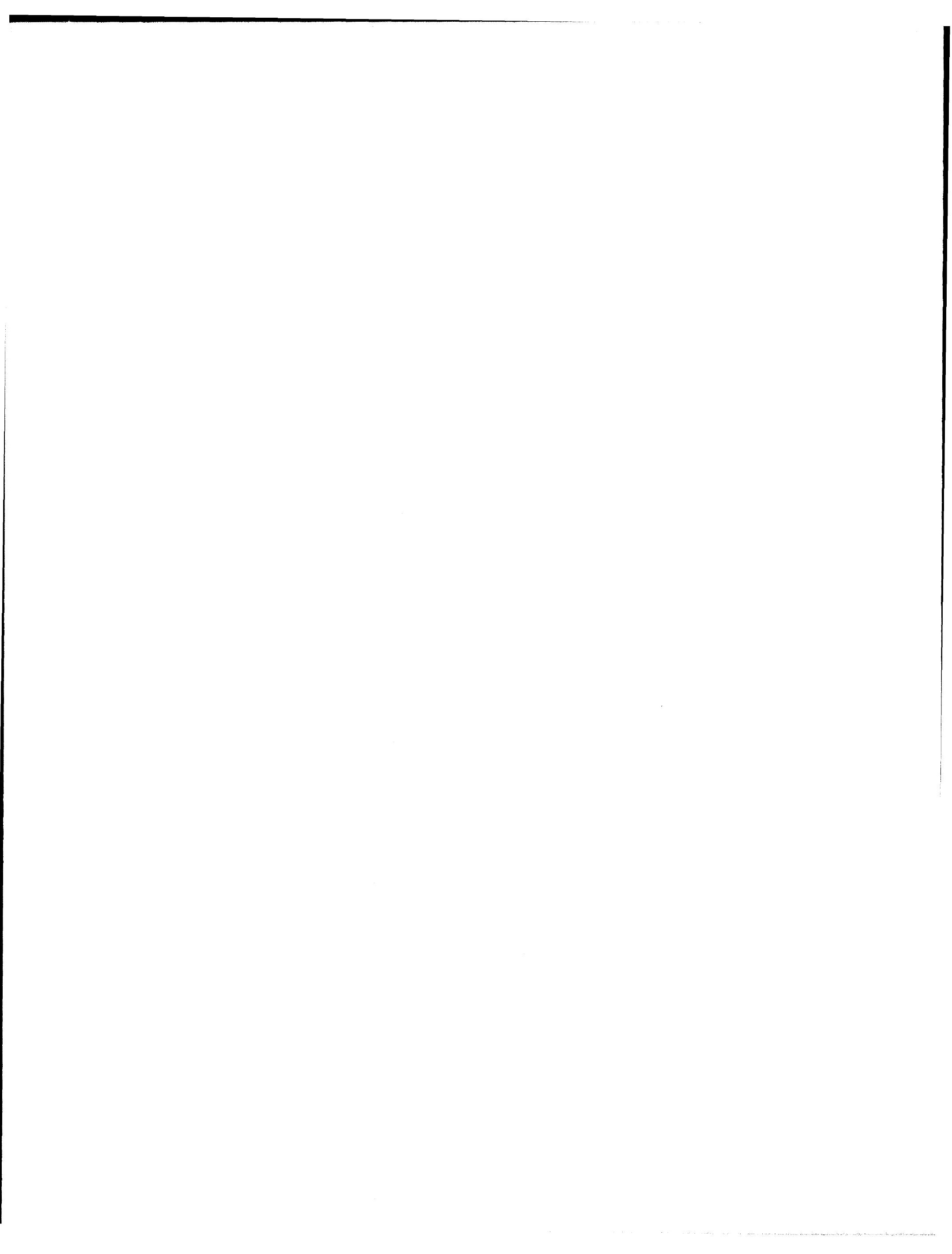

